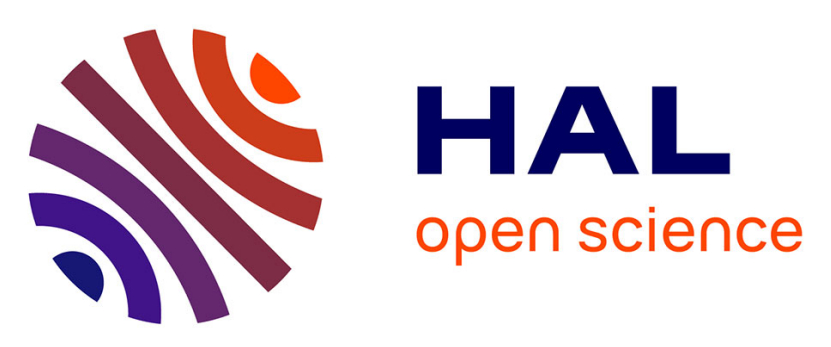

\title{
Patient-Specific Biomechanical Modeling of Cardiac Amyloidosis - A Case Study
}

Dominique Chapelle, Alessandro Felder, Radomir Chabiniok, Aziz Guellich, Jean-François Deux, Thibaud Damy

\section{To cite this version:}

Dominique Chapelle, Alessandro Felder, Radomir Chabiniok, Aziz Guellich, Jean-François Deux, et al.. Patient-Specific Biomechanical Modeling of Cardiac Amyloidosis - A Case Study. Functional Imaging and Modeling of the Heart 2015, Jun 2015, Maastricht, Netherlands. pp.295-303, 10.1007/978-3-31920309-6_34.hal-01174913

\section{HAL Id: hal-01174913 \\ https://inria.hal.science/hal-01174913}

Submitted on 10 Jul 2015

HAL is a multi-disciplinary open access archive for the deposit and dissemination of scientific research documents, whether they are published or not. The documents may come from teaching and research institutions in France or abroad, or from public or private research centers.
L'archive ouverte pluridisciplinaire HAL, est destinée au dépôt et à la diffusion de documents scientifiques de niveau recherche, publiés ou non, émanant des établissements d'enseignement et de recherche français ou étrangers, des laboratoires publics ou privés. 


\title{
Patient-specific biomechanical modeling of cardiac amyloidosis - A case study
}

\author{
D. Chapelle ${ }^{1}$, A. Felder ${ }^{1 \star}$, R. Chabiniok ${ }^{1,3}$, \\ A. Guellich ${ }^{2}$, J.-F. Deux ${ }^{2}$, T. Damy ${ }^{2}$

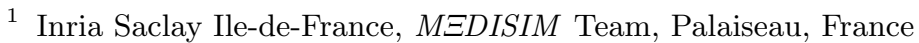 \\ 2 Henri Mondor Hospital, Créteil, France \\ ${ }^{3}$ St Thomas' Hospital, King's College London, UK
}

\begin{abstract}
We present a patient-specific biomechanical modeling framework and an initial case study for investigating cardiac amyloidosis (CA). Our patient-specific heartbeat simulations are in good agreement with the data, and our model calibration indicates that the major effect of $\mathrm{CA}$ in the biophysical behavior lies in a dramatic increase of the passive stiffness. We also conducted a preliminary trial for predicting the effects of pharmacological treatments - which is an important clinical challenge - based on the model combined with a simple venous return representation. This requires further investigation and validation, albeit provides some valuable preliminary insight.
\end{abstract}

Keywords: cardiac modeling; patient-specific; amyloidosis; heart failure

\section{Introduction}

Amyloidosis is a systemic disease with extracellular deposition of insoluble aggregates of beta-fibrillar proteins in various organs, leading to disturbances of normal tissue architecture and function [12]. Cardiac involvement is associated with poor prognosis. Morphological and functional abnormalities combine increase in cardiac wall thickness due to amyloid infiltration, and left ventricle (LV) diastolic and systolic dysfunction.

Global LV systolic function evaluated by ejection fraction is often preserved until advanced stages of the disease [4]. More recently, several studies have shown the ability of myocardial strain derived from echocardiography to detect subtle changes in systolic function in various diseases [6]. This approach seems to be useful in detecting cardiac involvement and was used to evaluate the prognosis of cardiac amyloidosis (CA) $[18,9,8]$. Moreover, magnetic resonance imaging (MRI) provides clinically valuable information beyond systolic function such as characterizing the myocardial tissue, and MRI late gadolinium enhancement (LGE) has been shown to be helpful for assessing the severity of cardiac amyloid deposition [16].

\footnotetext{
* Current affiliation: The Royal Veterinary College, London, UK
} 
Cardiological treatment of CA is also challenging as the usual drugs used in heart failure - such as those with negative inotropic and/or negative chronotropic effects (calcium channel blockers, beta-blockers) - are contraindicated. Furthermore, inotropic drugs may not be tolerated either due to the restrictive type of the cardiopathy.

Therefore, being able to formulate a biophysical model integrating the essential mechanisms of $\mathrm{CA}$ is expected to be valuable in order to better understand the pathophysiology of CA and the effect of drugs with different pharmacological properties. Moreover, setting up this type of model on a patient-specific basis [2] can be envisioned as a means to ultimately allow for optimizing therapeutic strategies based on predictive personalised modeling tools.

In the present article we sought to 1) build a personalised cardiac biophysical model of a patient with cardiac amyloidosis, 2) validate the model, 3) explore the effects of different pharmacological interventions using the model.

\section{Data}

Patient description - 72 year old man, with a CA due to a punctual mutation on TTR gene (Val122Ile). The patient gave his informed consent for the study.

MR imaging protocol and processing - Cardiac MRI was performed with a 1.5-T scanner (Magnetom Avanto, Siemens Healthcare). Contiguous short-axis sections encompassing the ventricles were acquired by the $\mathrm{k}$-space segmented Steady State Free Precession Cine sequence, with the following parameters: TR/TE, 2.8/1.4 (apparent TR, $31.4 \mathrm{~ms}$; 11 segments); flip angle, $82^{\circ}$; matrix size, $192 \times 192$; FOV, $300 \times 270 \mathrm{~mm}$; slice thickness, $8 \mathrm{~mm}$. Retrospective ECG gating was used with 25 phases per section. We then segmented the two ventricles in the end-diastolic images using the CardioViz3D software [17], and the Image Registration Toolkit (IRTK, under license from Ixico Ltd., see [13]) was used - with the motion tracking algorithm of [15] - to propagate the segmentation in all other time frames, hence, to compute LV volumes.

Echocardiography data acquisition and analysis - Echocardiography was performed using a Vivid 7 system (GE Vingmed). Data were analysed offline using the ECHOPac software (GE Healthcare). Acquisitions were digitally recorded at a high frame rate ( $>60$ frames/s) in three consecutive cycles. Longitudinal strain was computed from the standard LV apical views (2, 3 and 4 cavities) using 2D speckle tracking echocardiography analysis by automated function imaging (AFI, ECHO-Pac, GE). For strain processing, the region of interest (ROI) was automatically positioned to track the LV speckles frame-by-frame throughout the cardiac cycle. The endocardial contour and width were manually adjusted when necessary to provide optimal tracking. LV global (GLS) and regional longitudinal strain values of the 17 AHA classification segments were obtained. Average longitudinal strain was also calculated at the three LV levels (apical, mid-cavity and basal). 
Pressure measurements - The catheterization procedure was performed in the fasting state without sedation. Systemic blood pressure, central venous pressure, pulmonary artery pressure, and pulmonary capillary wedge pressure (PCWP) were determined over an average of 5 end-expiratory cardiac cycles, at steady state, with the patient in the supine position. The $0 \mathrm{mmHg}$ reference level was the mid-axillary line with the patient in the supine position. The pressure transducer was carefully maintained at the same level throughout the procedure. PCWP was assessed in West's zone 3, as verified by fluoroscopy, and found to be around $25 \mathrm{mmHg}$, a clear symptom of chronic heart failure.

The above 3 sets of data were acquired separately, albeit all within 24 hours, under the exact same medication, and with similar cardiac frequencies (around 80 bpms).

\section{Methods}

\subsection{Hierarchical modeling strategy}

We employ the same cardiac biomechanical model as in [1], for which the fundamentals have been presented and analysed in [3]. This model was also experimentally validated in [1], while some clinical validations of its predictive capabilities were given in [14].

As proposed in [1], in combination with a detailed 3D continuum mechanics model, we also consider a simplified - albeit consistent - model of the left ventricle. This simplified model is derived from the $3 \mathrm{D}$ model by assuming some spherical symmetry properties while retaining the full complexity of the constitutive properties, based on multi-scale considerations and compatible with the fundamental thermo-mechanical laws. Of course, spherical symmetry is not quite present in an actual left ventricle, but the resulting model - being in the form of a set of ordinary differential equations - is mostly used for fast simulation and calibration purposes. As explained in [1] we can very effectively calibrate the biophysical parameters using this simplified model, and then carry over the parameters to the 3D model in a straightforward manner.

\subsection{Personalisation strategy}
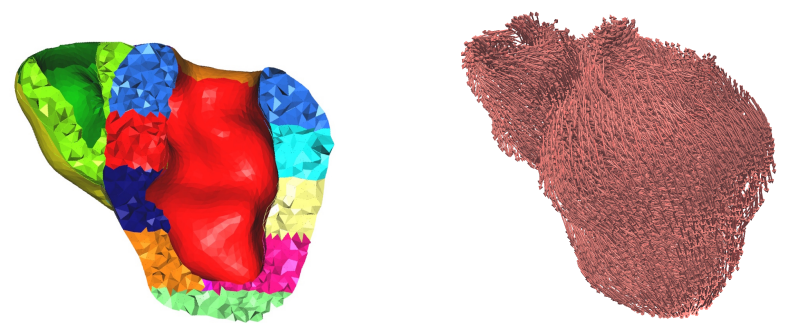

Fig. 1. Personalised anatomical model: AHA regions (left) and fiber directions (right) 
The patient-specific anatomical model was produced by using the segmentation of the end-systolic MR frame. The resulting surface mesh was further processed using the Yams software, and then the Ghs3D software was used to create a 3D tetrahedral mesh for a two-cavity (ventricular) model [5]. All the subsequent steps - for preparing the model and running simulations - were performed with the HeartLab software ${ }^{4}$. First, specific regions of interest were designated on the outer surfaces to provide for adequate boundary conditions [14], then the tetrahedral elements were tagged according to the AHA regions specifications. Furthermore, fiber directions were prescribed based on a given variation of the elevation angle across the thickness of the walls, see Fig. 1.

Concerning the personalisation of the biophysical parameters, in this preliminary study we did not resort to automatic estimation methods as in [2], and limited ourselves to - mostly global - manual calibration based on the simplified model and then carried over to the complete 3D model. Nevertheless, this calibration process is quite complex and requires some progressive systematic strategy as explained in [1].

\subsection{Venous return model}

A major challenge for this type of patient lies in determining an adequate therapeutic strategy - usually by a combination of drugs and pacing device specifically adapted to the patient in his/her current state, susceptible to quite rapid variations. Nevertheless, the actual cardiac state - e.g. as observed in the pressure-volume loop - is governed not only by the cardiac behavior coupled with the arterial response that determines the afterload, as accounted for in our above model, but also by the venous return that determines the preload. The latter is not included in our modeling components so far, as in fact we prescribed the preload. In order to account for variations in venous return as well, we use a description classically employed in cardiac physiology - valid for stationary states - that relies on total blood conservation written in the form [7]

$$
C_{v s} P_{v s}+C_{d} P_{d}=V_{\text {eff }},
$$

where $P_{d}$ and $P_{v s}$ respectively denote the distal arteries and venous system pressures, while $C_{d}$ and $C_{v s}$ denote the corresponding global elastance parameters, and $V_{\text {eff }}$ represents the total amount of blood that can fluctuate between the arterial and venous compartments. In addition, the distal flow between the arteries and veins is given by

$$
Q_{d}=\frac{P_{d}-P_{v s}}{R_{d}}
$$

where $R_{d}$ denotes the distal resistance. Of course, in a stationary regime this flow equals the cardiac output $Q$, hence we can use (1) to eliminate $P_{d}$ from (2) and obtain

$$
Q=\frac{V_{\mathrm{eff}}}{R_{d} C_{d}}-\left(1+\frac{C_{v s}}{C_{d}}\right) \frac{P_{v s}}{R_{d}},
$$

namely, a linear relationship between cardiac output and venous pressure.

\footnotetext{
${ }^{4}$ Proprietary software of Inria (main author P. Moireau)
} 


\section{Results}

\subsection{Model calibration and simulation results}
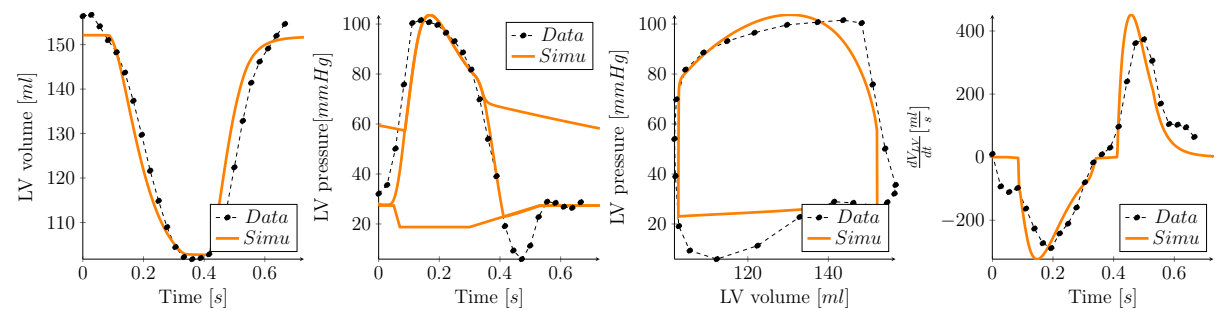

Fig. 2. Comparison of simulation results and data for LV. From left to right: volume; pressure; PV-diagram; outgoing flow. Black dots correspond to MR frames, registered with pressure recordings based on R-waves of ECGs
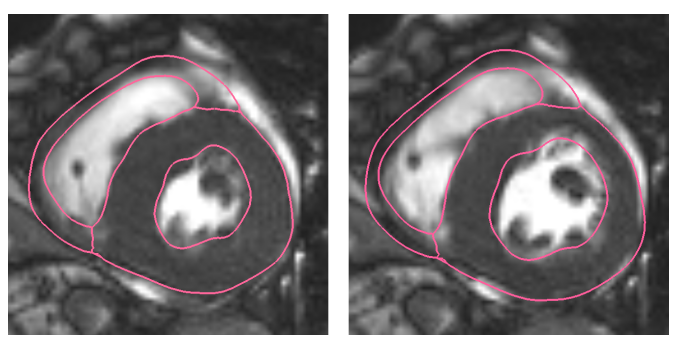

Fig. 3. Comparison of simulated mesh contours with MR data at end-systole (left) and end-diastole (right)

Compared with previous modeling trials, our calibration here was guided by clinical evidence that amyloidosis results into a stiffer passive behavior, while the slope of the end-systolic pressure-volume relationship (ESPVR) is roughly preserved - albeit shifted towards large volumes [11]. As a consequence, we increased the passive coefficients without changing the contractility parameter - compared to a normal heart - while adjusting the Frank-Starling function to operate with larger volumes, see [1] for a definition of this function. We show in Fig. 2 the resulting LV pressure, volume and flow indicators computed with the 3D model and compared with the processed data. We also show in Fig. 3 a comparison of the model boundaries with MRI in short axis view at end-systole and end-diastole. The calibrated biophysical parameters are given in Table 1, compared with those calibrated in [1] - in the same units - for a normal heart. As we immediately observe here, the main difference in these parameters concerns 
the passive law, for which an increase by a factor 20 or so - for the main two parameters $\left(C_{0}, C_{2}\right)$ - was found to be consistent with the data.

Table 1. Comparison of CA patient-specific model parameters with normal heart from [1]. $\left(C_{0}, C_{2}\right)$ and $\left(C_{1}, C_{3}\right)$ denote elastic parameters, $\eta$ viscous modulus, $\sigma_{0}$ contractility (active behavior), $\left(R_{p}, C_{p}\right)$ and $\left(R_{d}, C_{d}\right)$ Windkessel parameters (proximal and distal)

\begin{tabular}{c|cccccccc} 
& $C_{0}, C_{2}$ & $C_{1}, C_{3}$ & $\sigma_{0}$ & $R_{p}$ & $C_{p}$ & $R_{d}$ & $C_{d}$ & $\eta$ \\
\hline $\mathrm{CA}$ & $1.110^{5}$ & $1.110^{-1}$ & $4.210^{5}$ & $1.810^{7}$ & $5.610^{-10}$ & $1.110^{8}$ & $210^{-8}$ & 560 \\
Normal & $5.710^{3}$ & $1.110^{-1}$ & $6.210^{5}$ & $210^{7}$ & $210^{-9}$ & $210^{8}$ & $0.510^{-8}$ & 70
\end{tabular}
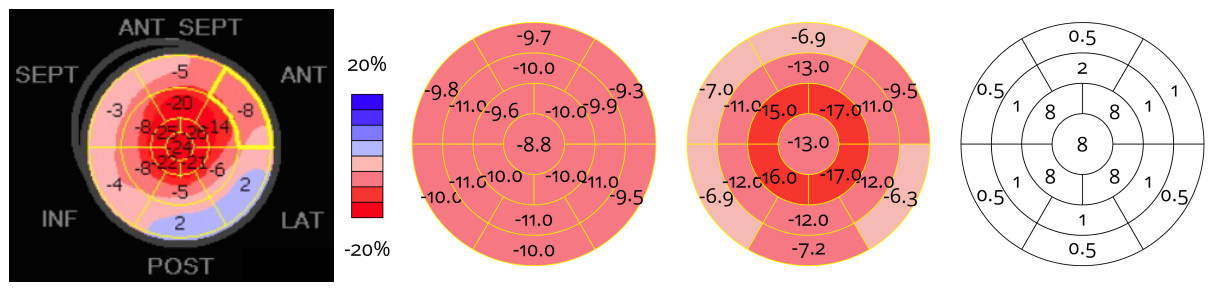

Fig. 4. Comparison of simulation results and processed data for peak longitudinal systolic strain in AHA regions. From left to right: data (AHA region \#1 surrounded in bold yellow); simulation with uniform contractility; with non-uniform contractility; non-uniform contractility scaling coefficients

Having at our disposal peak longitudinal strain data in AHA regions, we proceeded further in the calibration by allowing the contractility parameter to vary over these regions. We can thus account for the non-uniform deposition of amyloid fibrils in the myocardium, with a much higher density near the base [10]. We accordingly prescribed a varying contractility by applying a scaling coefficient in the AHA regions. This is shown in Fig. 4 with the resulting longitudinal strains averaged within each AHA region, and compared with the data.

\subsection{Prediction of patient states}

We now want to conduct a preliminary study of the predictive capabilities of our modeling, using the simplified cardiac model presented above together with the venous return relation (3). Assuming a fixed difference between the preload and the venous return - prescribed to $7.5 \mathrm{mmHg}$ in our simulations, in accordance with our pressure data - the cardiac function itself determines another relation between the venous pressure and the output, via the Frank-Starling mechanism. In fact, we can very effectively use our above simplified model to characterize this relation by running simulations with a given preload until the cardiac cycle stabilizes. The current cardiac state is then summarized by the intersection of the two curves. We plot these curves for our patient in Fig. 5-left, compared with 
those obtained for normal hearts. In our case, we inferred the venous return curve (3) by observing that the maximum flow equals $V_{\text {eff }} / R_{d} C_{d}$, where there is no reason to believe that $V_{\text {eff }}$ differs from a physiological value. Subsequently, as the operating point is known - from the data - for the patient we can draw the whole line, and infer $C_{v s}$.
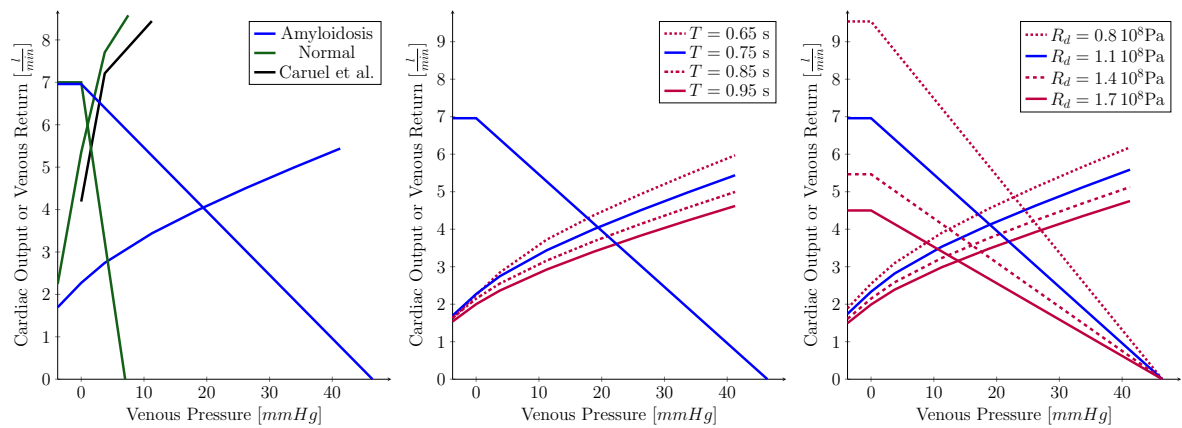

Fig. 5. Computed cardiac function and venous return curves. From left to right: patient in baseline compared to normal heart in [7] (green) and [1] (black); rhythm variations ( $T$ denotes beat period in s); systemic resistance variations

With this simplified - albeit global and personalized - model of the cardiovascular system we can investigate the effect of various drugs on the patient. Clearly, judging from the differences in 1) cardiac states, and 2) slopes of the cardiac and venous functions in Fig. 5-left, we can presume that the patient's responses will be very different from those of a healthy individual. In Fig. 5-center we show the effect of varying the heart rhythm, which can be produced by using beta-blockers, in particular. In our patient's case, lowering the rhythm - an effect of beta-blockers - would decrease the output and increase the - already dangerously high - venous pressure, hence this should be avoided. In Fig. 5-right we display the effect of varying the systemic resistance, as could be obtained with ACE-inhibitors. The effect is more complex, as both cardiac function and venous return curves are affected by this parameter. We see that a decrease in systemic resistance - as induced by ACE-inhibitors - would improve the output, but again at the cost of increasing the venous pressure, quite importantly in fact due to the low slope of the cardiac curve.

\section{Discussion}

Concerning the numerical simulations of cardiac cycles, the main difference appearing in Fig. 2 in the global indicators between the numerical results and the data pertains to the initial part of the rapid filling phase, during which the measured LV pressure decreases significantly below the simulated value. In fact, 
we know from the ECG that the patient suffers from a strong delay in atrioventricular conduction, hence, during that initial filling phase the left atrium is already contracting. Therefore, the simulation-data discrepancy can only be due to a strong heterogeneity between venous, atrial and ventricular pressures during that phase, whereas our model is designed to instead equilibrate these pressures. This could be changed, but we do not consider this improvement as very important from the point of view of the overall cardiac function.

Strain results obtained with varying contractility have already shown some good adequacy with the data, recall Fig. 4. In order to obtain more accurate results, a more detailed estimation of the parameter values would be needed, see below.

\section{Conclusions}

We have presented a patient-specific modeling framework and an initial case study for investigating cardiac amyloidosis. Our patient-specific heartbeat simulations show very good agreement with the data as regards the pressure-volume indicators, and reasonable agreement with the strain measurements when allowing the physical parameters to spatially vary. Better accuracy in the strains would require resorting to automatic parameter estimation as in [2], which is a natural perspective of this study.

We also conducted a preliminary trial for predicting the effects of pharmacological treatments on the patient based on the model. This of course requires further investigation and validation, including with retrospective assessments.

Our case study indicates that the major effect of CA in the biophysical behavior lies in a dramatic increase of the passive stiffness. This needs to be confirmed by including other similar cases in a further study, albeit provides some valuable preliminary insight. Also, beyond the specific type of CA considered here - quite important in terms of prevalence with $4 \%$ of African-American people carrying such Val122Ile gene mutations - other types of CA warrant similar investigations.

Acknowledgments: The authors are very grateful to Philippe Moireau for valuable discussions and for the use of his numerical simulation software HeartLab, and also wish to thank Gabriel Valdes Alonzo (intern) for some helpful numerical verifications.

\section{References}

1. M. Caruel et al. Dimensional reductions of a cardiac model for effective validation and calibration. Biomech. Model. Mechan., 13(4):897-914, 2014.

2. R. Chabiniok et al. Estimation of tissue contractility from cardiac cine-MRI using a biomechanical heart model. Biomech. Model. Mechanobiol., 11(5):609-630, 2012.

3. D. Chapelle et al. Energy-preserving muscle tissue model: formulation and compatible discretizations. Int. J. Multiscale Com., 10(2):189-211, 2012. 
4. T. Damy et al. Role of natriuretic peptide to predict cardiac abnormalities in patients with hereditary transthyretin amyloidosis. Amyloid, 20(4):212-220, 2013.

5. P. Frey and P.L. George. Mesh Generation. Wiley, 2nd edition, 2008.

6. L. Gray Gilstrap et al. Predictors of survival to orthotopic heart transplant in patients with light chain amyloidosis. J. Heart Lung Transpl., 33(2):149-156, 2014.

7. B.M. Koeppen and B.A. Stanton. Berne and Levy Physiology. Mosby, 6th updated edition, 2009.

8. J. Koyama and R.H. Falk. Prognostic significance of strain Doppler imaging in light-chain amyloidosis. J. Am. Coll. Cardiol. Img., 3(4):333-342, 2010.

9. J. Koyama et al. Longitudinal myocardial function assessed by tissue velocity, strain, and strain rate tissue Doppler echocardiography in patients with AL (primary) cardiac amyloidosis. Circulation, 107(19):2446-2452, 2003.

10. D. Liu et al. Echocardiographic evaluation of systolic and diastolic function in patients with cardiac amyloidosis. Am. J. Cardiol., 108(4):591-8, 2011.

11. R.E. McCarthy and E.K. Kasper. A review of the amyloidoses that infiltrate the heart. Clinical Cardiology, 21(8):547-52, 1998.

12. E. Roig et al. Outcomes of heart transplantation for cardiac amyloidosis: Subanalysis of the Spanish registry for heart transplantation. Am. J. Transplant., 9(6):1414-1419, 2009.

13. D. Rueckert et al. Nonrigid registration using free-form deformations: Application to breast MR images. IEEE Trans. Med. Imaging, 18(8):712-721, 1999.

14. M. Sermesant et al. Patient-specific electromechanical models of the heart for the prediction of pacing acute effects in CRT: A preliminary clinical validation. Med. Image Anal., 16(1):201-215, 2012.

15. W. Shi et al. A comprehensive cardiac motion estimation framework using both untagged and 3D tagged MR images based on non-rigid registration. IEEE Trans. Med. Imaging, 31(6):1263-1275, 2012.

16. I.S. Syed et al. Role of cardiac magnetic resonance imaging in the detection of cardiac amyloidosis. J. Am. Coll. Cardiol. Img., 3(2):155-164, 2010.

17. N. Toussaint et al. An integrated platform for dynamic cardiac simulation and image processing: Application to personalised tetralogy of Fallot simulation. In Proc. Eurographics Workshop on Visual Computing for Biomedicine, 2008.

18. C.S. Vikram. Removing the diffraction halo effect in speckle photography of sinusoidal vibration. Appl. Opt., 29(25):3572-3573, 1990. 